\title{
LA ASISTENCIA SOCIAL VISTA POR LOS BENEFICIARIOS DE UN PROGRAMA \\ DE LUCHA CONTRA LA POBREZA: APROPIACIÓN Y CONSTRUCCIÓN DE REPRESENTACIONES ALREDEDOR DEL PROGRAMA FAMILIAS EN ACCIÓN
}

\author{
Valeria Andrea Ayola Betancourt ${ }^{*}$
}

\section{Resumen}

Este artículo explora la construcción de representaciones y las dinámicas de apropiación del programa Familias Acción por parte de sus beneficiarios. El enfoque cognitivo de políticas públicas nos permite estudiar el instrumento en tanto portador de marcos interpretativos de regulación de lo social susceptibles de ser transmitidos a los actores involucrados. En este se describe la manera como los beneficiarios son sujetos tanto receptores como interpretativos de la intervención estatal, y algunos efectos sociales derivados de la puesta en marcha de los programas teniendo en cuenta el ámbito local. Para esto, se privilegia una metodología cualitativa de análisis y la entrevista semidirectiva como técnica de investigación. Nuestros resultados se sustentan en un trabajo de campo, el cual, de manera comparativa, fue realizado en medio rural y urbano en el departamento de Bolívar.

Palabras clave: asistencia social, política social, pobreza, Familias en Acción, instrumentos, transferencias monetarias condicionadas.

\footnotetext{
* Magíster en Investigación en Ciencias Sociales, mención estudios políticos, Escuela de Altos Estudios en Ciencias Sociales (eHEss); Magíster 1 en Economía Aplicada, Université d'Aix-Marseille. Actualmente inscrita en un año preparatorio al doctorado en Sociología en el EHess, París (Francia).valeria.ayola@ehess.fr

Recibido: 19 de marzo de 2016 / Modificado: 3 de mayo de 2016 / Aceptado: 6 de mayo de 2016

Para citar este artículo

Ayola Betancourt, V. A. (2016). La asistencia social vista por los beneficiarios de un programa de lucha contra la pobreza: apropiación y construcción de representaciones alrededor del programa Familias en Acción. OPERA, 18, pp. 85-104. DOI: http://dx.doi.org/10.18601/16578651.n18.06
} 
A COGNITIVE APPROACH TO SOCIAL ASSISTANCE INSTRUMENTS: HOW PUBLIC ASSISTANCE RECIPIENTS REPRESENT AND INTERPRET THE ANTI-POVERTY PROGRAM FAMILIAS EN ACCIÓN

\section{Abstract}

This article considers the contribution of the cognitive framework of public policy and the instrument approach to understanding poverty regulation in Colombia through its instruments. We analyze the relationship between the ideological frame of the Familias en Acción program and the recipient's construction of representations, meanings and interpretations about it. It describes the manner in which the beneficiaries are interpreting the State intervention, but it also describes some social effects arising from the implementation of the programs, taking into account the local scope. For this we use qualitative research techniques and stand out the semi-structured interview as method. Our fieldwork, is based on a comparison between Cartagena city and the San Jacinto's rural zone.

Key words: Fight against poverty, social assistance, Familias en Acción, instruments, conditional cash transfers.

\section{INTRODUCCIÓN}

La manera en que las sociedades han buscado regular las formas extremas de diferenciación entre sus miembros ha cambiado según los órdenes morales, políticos y sociales existentes. Asimismo, la responsabilidad de atender a los pobres de una sociedad ha sido asignada a diferentes instituciones dentro de las cuales podemos mencionar el clan, la familia, la comunidad, la iglesia o el Estado, hasta llegar al escenario actual donde esta se vuelve un asunto que se extiende más allá de las fronteras nacionales.

De la mano de las instituciones financieras y las organizaciones multilaterales, la "lucha contra la pobreza" (LCP) logra tematizarse en la arena internacional como asunto prioritario de los países en desarrollo (Gabas y Laporte, 2012). Lo anterior obedece a un cambio de enfoque que tiene lugar en los ańos noventa, donde se reconocen los efectos regresivos de las medidas de ajuste estructural ${ }^{1}$. Siguiendo este cambio, propulsar el crecimiento económico de una nación sigue siendo la principal estrategia de LCP pero se reconoce que para esto los Estados deben implementar acciones específicas destinadas a combatirla.

Los programas de transferencias condicionadas (PTC), amparados en un discurso eco-

1 En 1988, se realiza un simposio donde se discute el impacto del ajuste para los pobres, el documento Targeted programs for the poor during structural adjustment concentra parcialmente esta discusión. 
nómico que postula su pertinencia y eficiencia (Rawlings, 2004), han logrado posicionarse como instrumentos "modelos" de esta lucha. Siendo su difusión tal (Debonneville y Díaz, 2011), que estos han sido implementados en al menos treinta países del mundo, de los cuales dieciocho pertenecen a América Latina ${ }^{2}$.

Abordar el tema de los instrumentos de lucha contra la pobreza nos exige, entonces, tener en cuenta la dimensión global que condiciona las políticas sectoriales nacionales. El objetivo de este artículo no es el de mostrar cómo opera la difusión y circulación de instrumentos a nivel internacional, tampoco el de estudiar los resultados o impactos ligados a la puesta en marcha del instrumento en el caso colombiano. Por el contrario, nos hemos propuesto estudiar la manera en que los instrumentos de política pública -en este caso el PTC Familias en Acción- ${ }^{3}$ son portadores de marcos interpretativos formulados a nivel macro, susceptibles de ser transmitidos y aprehendidos o reinterpretados a nivel micro por los destinatarios de dichos programas.

Nos interesa estudiar lo anterior teniendo como objeto de estudio la política de asistencia social. Esta, por un lado, refleja la manera en que las sociedades se piensan a sí mismas y buscan regular la pobreza a través de formas institucionalizadas. Por otro lado, nos interesa estudiar el instrumento mencionado como acción que emana desde el poder político e ideológico hacia la sociedad. Teniendo en cuenta lo anterior, se estudia la política de asistencia social como elemento interpretativo de lo político-social en la medida que define categorías entre aquellos que son susceptibles de recibir la asistencia, las condiciones que estos deben cumplir, los derechos susceptibles de reivindicar e incluso el comportamiento moral que se debe adoptar (Giraldo, 2013).

Estudiar la relación de asistencia social a través de los instrumentos de política pública nos permite, de este modo, entender la manera como se estructura el orden social de la mano del poder político. La asistencia es una relación que define la posición social de quien la recibe, ubicándolo por lo general en el último estrato de la sociedad (Simmel, 2011).

Adoptar esta perspectiva de análisis implica superar los análisis ya tradicionales en el tema, los cuales han buscado, desde un pretendido positivismo, medir las variables del programa desde perspectivas tecnocráticas; esto, siguiendo el argumento de André NoëlRoth (2009), poco ha contribuido a dinamizar el debate sobre la política social o al fortalecimiento de la democracia en Colombia.

2 En 1998, la lucha contra la pobreza será adoptada por los gobiernos latinoamericanos en la cumbre de las américas realizada en Santiago de Chile.

3 En Colombia, el PTC Familias en Acción ingresa como parte de la red de apoyo social del Plan Colombia en el año 2001, este verá importantes variaciones a través de los años, entre las que se puede mencionar el aumento del presupuesto asignado y de la cobertura. Este programa se consolida en el marco jurídico colombiano con la aprobación de la Ley 1532 de 2012, la cual nos confirma la inserción del programa en el sistema normativo colombiano, motivándonos a estudiarlo con mucha más profundidad. 
En esta misma línea, nos interesa mencionar como puntos de partida analíticos del presente documento, el estudio comparativo sobre la pertinencia del programa como política social para América Latina emprendido por Bruno Lautier (2010, 2013); la propuesta que desde el caso argentino logra poner a prueba la pertinencia del discurso económico que sustenta la difusión de las políticas contra la pobreza (Lo Vuolo et al., 2004), y el enfoque de la Escuela para el Desarrollo, la cual ha buscado, desde una perspectiva holista, explicar el cambio de modelo social que significó para Colombia el paso de una estrategia de tipo bismarckiana a una centrada en la focalización (Giraldo, 2013).

\section{EL ENFOQUE COGNITIVO DE POLÍTICAS PÚBLICAS Y EL ENFOQUE POR LOS INSTRUMENTOS}

El enfoque cognitivo de políticas públicas que encuentra en Pierre Muller (2000) su principal exponente, nos permite ampliar el objeto de estudio de la disciplina de las políticas públicas a la relación que existe entre las políticas y la construcción de un orden sociopolítico. Este nos permite integrar la dimensión de lo global a través del estudio de la articulación entre referentes sectoriales y globales. El concepto de referencial nos habla de la existencia de marcos interpretativos de lo real, los cuales influencian los modelos normativos de acción al ser reconocidos como la "verdad" por una mayoría de actores involucrados en la implementación y evaluación de los instrumentos de política pública.
El Estado-nación, para Muller (2000), ha dejado de ser el lugar donde se construyen referentes sectoriales de interpretación del mundo. Los gobiernos deben adaptarse a marcos de interpretación construidos más allá de sus fronteras y, por tanto, a un referente global valorizado. El referencial actual se encuentra determinado por el giro neoliberal experimentado a finales del siglo xx (Jobert, 1994) y por los nuevos modos de gobernanza internacional, donde una multiplicidad de actores como Estados-nación, organizaciones multilaterales, instituciones económicas, sector solidario o sector privado confluyen.

Bajo este enfoque interpretamos la política social en su articulación con el referente global de mercado, el cual no es solo portador de un marco interpretativo del mundo, sino que también contiene una definición de orden social.

Por otro lado, el enfoque por los instrumentos nos permite estudiar la política social desde su materialidad, pues este parte del instrumento - programas, proyectos, políticas sectoriales- como variable explicativa del cambio (Lascoumes y Simard, 2011). Bajo este enfoque, los instrumentos son vistos como "instituciones sociológicas" (Hood, citado en Lascoumes y Simard, 2011) portadores de valores, pocas veces axiológicamente neutros y alimentados de una interpretación de lo social o concepciones precisas del modo de regulación considerado (Lascoumes y Le Galès, 2004, p. 13).

Este enfoque puede ser puesto en práctica a través del estudio de la construcción y adaptación de las herramientas consideradas 
en el contexto local, pero también de los efectos sociales que estas generan en los actores implicados, como por ejemplo, de las dinámicas de apropiación (creencias, estrategias, representaciones $)^{4}$.

A partir del concepto de instrumento, algunas preguntas orientan el presente documento: ¿cuáles son los valores transmitidos por el instrumento privilegiado de lucha contra la pobreza en el país? ¿De qué manera el instrumento es portador de significaciones y representaciones susceptibles de ser asimiladas por los destinatarios?

\section{METODOLOGÍA}

Este documento es una prolongación de la investigación realizada sobre la regulación de la pobreza y el vínculo de ciudadanía en el departamento de Bolívar entre 2014 y 2015. El presente documento se sustenta así en una inmersión en campo que se realizó a principios del año 2015 en el distrito de Cartagena de Indias y en los municipios de Santa Catalina y San Jacinto en el departamento de Bolívar; estos dos últimos fueron escogidos basándonos en el índice de necesidades básicas insatisfechas (NBI) como referente de la situación global de pobreza.

La metodología utilizada fue de tipo cualitativo, con representación en medio rural y urbano ${ }^{5}$; se privilegió la entrevista semiestructurada como técnica, la cual, siguiendo a Pinson y Sala-Pala (2007), constituye una herramienta pertinente para quien se interese en el estudio de la acción pública en su dimensión sincrónica o histórica. Esta, más allá de su uso informativo, puede ser movilizada para un uso comprensivo que involucre el análisis de las prácticas cotidianas y de las representaciones que las orientan.

Con esto nos interesamos en el estudio de los discursos como productos sociales orientadores de prácticas políticas, lo que nos permite superar el análisis del discurso en ciencia política, analizado principalmente desde su componente ideológico.

Así las cosas, se entrevistaron cuatro funcionarios ${ }^{6}$ directamente relacionados con la operación local del programa y 40 beneficia-

\footnotetext{
4 La noción de representación social fue tratada por los fundadores de la sociología como Durkheim y Weber, esta será retomada en los años sesenta por la psicología social y hoy en día es considerado un concepto por profundizar en las ciencias sociales. Las representaciones sociales nos hablan de una forma de conocimiento específica, la del sentido común; estas tienen valor de realidad concreta y orientan las conductas de los individuos. Jodelet (1989) las define como un proceso mediante el cual el individuo se apropia pero también reconstruye su medio ambiente externo.

5 El trabajo de campo en el distrito de Cartagena incluyó visitas y entrevistas a habitantes de los barrios La Candelaria, Vista Hermosa, Henequén, Torices, Isla de Loba/Pozón y Villas de Aranjuez. El componente rural incluye las veredas de Arenas, Charquitas, San Cristobal, Paraíso. Como rural/urbano los barrios San José, La paz, Villamaría de San Jacinto, así como el barrio San José de Santa Catalina.

6 Teniendo en cuenta que estos son fácilmente identificables, por un acuerdo de confidencialidad los discursos de estos funcionarios no serán publicados.
} 
rios del programa de los cuales 7 eran madres líderes ${ }^{7}$. Estas entrevistas fueron realizadas directamente por el sujeto investigador en los lugares de residencia o de paso habitual de quienes componían la muestra. Con los beneficiarios se realizaron cuatro grupos focales, veinticinco entrevistas semiestructuradas ${ }^{8}$ y dos entrevistas abiertas. La información socioprofesional de los entrevistados quedó consignada en una base de datos y las observaciones en un diario de campo.

El contenido de las entrevistas fue analizado teniendo en cuenta los aportes del Análisis Crítico del Discurso (ACD), el cual nos invita a ir más allá del análisis textual y contextual de las estructuras discursivas para tener en cuenta los sentidos implícitos y latentes de los discursos (Sánchez, 2004). Lo anterior nos permitió identificar estructuras de representaciones que dan cuenta de la existencia de una diversidad en los niveles explicativos y de conductas que se derivan de la participación de los beneficiarios en el programa.

Para este documento, se buscó contrastar los conceptos y fundamentos teóricos que sostienen los PTC, los cuales serán descritos en la sección siguiente con la información empírica recopilada a través de las entrevistas semiestructuradas, información que se verá plasmada en la última sección.

\section{EL ANDAMIAJE TEÓRICO DE LOS PROGRAMAS DE TRANSFERENCIAS CONDICIONADAS}

Los PTC representan un nuevo saberhacer en el tratamiento de la pobreza, estos combinan los conceptos de pobreza ligados a la libertad, de Amartya Sen; la idea del capital humano aplicado al desarrollo; el manejo social del riesgo, y, por último, la idea de focalización. Cada una de estas constituyen las tecnologías de intervención que Álvarez (2013) denominaría partes constitutivas de la nueva economía política de la pobreza, lo cual se puede interpretar como un marco ideológico. En esta sección examinaremos brevemente estos conceptos para luego relacionarlos con nuestros resultados más claramente.

Los PTC encierran una interpretación causal y una normativa de la pobreza. De acuerdo con la primera, esta es causada por fallas de mercado que no permiten que toda la

De acuerdo al manual operativo del programa del 2008 "La madre líder es una representante de las familias beneficiarias elegida entre las madres titulares con el fin de representarlas en todos los aspectos relacionados [...]. La labor de la madre líder es voluntaria, solidaria y no remunerada. Para ser madre líder se requiere saber leer y escribir, tener vocación de trabajo comunitario, ser beneficiaria directa y cumplir con todos los requisitos establecidos en los tiempos y condiciones que determina el Programa”.

8 Se buscó controlar el riesgo de uso de estrategias discursivas por parte de los entrevistados ante la presencia de personas externas, como lo es el investigador; en un ejercicio de reflexión, se buscó minimizar este riesgo al hacer énfasis en la neutralidad de la investigación, y en la no existencia de vínculos con la administración pública o con el poder político. También se procuró realizar las entrevistas en una dinámica más cercana a la observación participante integrando los espacios habituales de interacción y socialización. 
población pueda beneficiarse del crecimiento económico, una debilidad en la dotación de factores de producción y de capital -incluido el capital humano-, pero también debido a choques transitorios como las crisis económicas. Por su parte, la interpretación normativa nos dice que es necesario mitigar la pobreza sin desmontar el conjunto de medidas económicas que conducen al desarrollo económico de un país y las cuales fueron plasmadas en el conjunto de medidas del denominado Consenso de Washington?.

Los PTC son, de esta forma, considerados herramientas eficientes, técnicas y por tanto neutras de la LCP. De acuerdo a sus defensores, estos son programas de sustento a la demanda que corrigen los resultados desiguales de los intercambios de mercado, al mismo tiempo que son generadores de externalidades positivas (Rawlings, 2004). Considerando como un a priori la escasez de recursos en los países en vía de desarrollo, focalizar se vuelve la prueba de la eficiencia de estos instrumentos ya que de esta forma los recursos públicos se centran solo donde son más productivos, es decir, en los más pobres de los pobres, en detrimento de las soluciones universalistas consideradas costosas. Esto es a su vez una forma de prevenir la aparición de la figura del free-rider, una de las principales preocupaciones de la economía neoliberal.
Las transferencias se presentan como políticas de desarrollo y, a la vez, como integrantes de la política social (Merrien, 2013). A partir de la década del 2000, la protección social entra a ser definida por la doctrina del manejo social del riesgo, la cual se basa en el manejo del riesgo futuro del ingreso, óptica bajo la cual los individuos previenen, mitigan y se recuperan del riesgo vía el mercado asegurador (Holzmann y Jorgensen, 2000). La excepción son entonces los más pobres, para los cuales se conciben mecanismos público-privados de protección del riesgo. Cabe mencionar que las transferencias inicialmente fueron concebidas como estrategias de recuperación ante choques, a pesar de esto la experiencia latinoamericana nos indica que estas se han vuelto estrategias permanentes de prevención y mitigación.

Ahora bien, de acuerdo con los aportes de Amartya Sen, la pobreza debe ser medida teniendo en cuenta no los recursos, sino el conjunto de capacidades de los individuos que les permitan su transformación en los funcionamientos que estos valoricen. Las inversiones en capital humano de los PTC son vistas como una manera de propulsar dichas capacidades.

Teniendo como referente el concepto de trampas de pobreza, para la economía del desarrollo la condición de pobreza se expli-

9 Término acuñado por el economista John Williamson para explicar el conjunto de medidas de política económica de corte neoliberal aplicadas a partir de los ańos ochenta como salida impuesta por el FмI y el вм a los países del sur ante el estallido de la crisis de la deuda externa, donde podemos resaltar privatizaciones, modernización estatal, entrada de inversión extranjera, desmonte de aranceles, disciplina fiscal, etc. 
ca por una insuficiente inversión en capital humano ${ }^{10}$, lo que impide que los pobres logren ser productivos y competitivos. Los PTC buscan aumentar el stock de capital humano atacando la deserción escolar y el insuficiente uso de las estructuras de salud; de esta forma, se busca incidir sobre la demanda del servicio, mas no sobre la oferta de este (la cual se presume dada), generando que la responsabilidad se traslade del Estado hacia el hogar y el individuo.

Por otro lado, para Sen (1999), la capacidad de elección es un componente esencial que permite el empoderamiento $y$, por consiguiente, el aumento de libertad de las personas. Basados en esto, los PTC permiten el libre uso de las transferencias como una manera de aumentar la capacidad de decisión y autonomía de los hogares pobres, lo cual los ha hecho alejarse de los programas de activación (workfare) que exigen la vinculación laboral como contrapartida a las ayudas monetarias ${ }^{11}$.

No obstante lo anterior, aun cuando se exalta la libertad de agencia al permitir el libre uso de la transferencia, los programas establecen unas condicionalidades. De hecho, los PTC tienen como principio la incitación (método bastante utilizado por los ideólogos de la teoría de juegos y del rational choice), la cual busca generar cambios comportamentales y decisiones finales más eficientes, al transformarlas en un cálculo costo-beneficio (Morley y Codey, 2003).

Por su parte, la rama de la economía del desarrollo que se sustenta en experiencias empíricas, ha profundizado en los impactos económicos del comportamiento de los pobres. Para Banerjee y Duflo (2012), las decisiones erradas que los pobres toman -como por ejemplo, abandonar la escuela o no sanarse- se explican no solo por sus limitadas dotaciones de base sino por comportamientos como la procastinación, un elevado sentido de lo inmediato, así como una subestimación de los beneficios reales. Por esta razón, la incitación resulta el método más adecuado para lograr los objetivos de reducción de la pobreza (Banerjee y Duflo, 2012, pp. 70, 93).

Se trata, entonces, de una visión normativa de los pobres, la cual renueva viejas representaciones acerca de estos. Lo anterior, siguiendo a Álvarez (2013), contribuye a reforzar el prejuicio tutelar que consiste en explicar la pobreza como un asunto relacionado con la carencia de valores en los pobres (como proclives a no educarse o a no cuidar de su salud) y, por tanto, como una cuestión de tipo individual o familiar.

10 El capital humano es considerado, desde los trabajos de Becker (1964), un tipo de capital como cualquier otro, pero será con los diferentes trabajos de Romer y Barro en los años noventa, que la inversión en capital humano pasa a considerarse determinante en la función de producción de la economía, y, en esta misma línea, establece una correlación entre esta y el crecimiento económico.

11 A pesar de esto, los bajos montos manejados por las transferencias han tenido como finalidad el no generar incentivos contrarios a la inserción laboral, uno de los puntos más debatidos por los académicos de LCP. 


\section{RESULTADOS DE UN ESTUDIO COMPARATIVO EN EL DEPARTAMENTO DE BOLÍVAR}

Antes de describir los resultados relacionados con el objeto de este artículo, resulta conveniente mencionar los resultados más importantes de la investigación que da origen a este documento (Ayola, 2015). Dicha investigación concluye que el objetivo de romper la transmisión intergeneracional de la pobreza se encuentra más allá del alcance del programa, ya que la dependencia económica de los hogares respecto a la transferencia es alta pues la integración de estos al mercado laboral está sujeta al mercado informal y los bajos montos no permiten la inversión en activos productivos.

En cuanto al componente de integración, el programa ha permitido una integración social de los beneficiarios con su grupo de referencia (familia, vecinos, comunidad) mas no con el resto de la sociedad. La integración política tampoco ha tenido lugar, pues los encuentros y las opciones de participación en el marco del programa no constituyen opciones reales de participación que impliquen una transmisión de poder de decisión en favor de los beneficiarios ni de los pobres en general.

Por último, el resultado que nos interesa señalar es que el impacto del programa puede encontrar importantes variaciones en función de la condición y posición social ${ }^{12}$ de los ho- gares. Los resultados obtenidos nos indican que el programa se acerca a sus objetivos de mitigar la transmisión intergeneracional de la pobreza en aquellos individuos y hogares que presentan una menor dependencia económica y que, por tanto, se encuentran más cerca de la línea de la pobreza.

La heterogeneidad de los pobres fue analizada con base en la información socioprofesional consignada en la base de datos y las informaciones recopiladas en las entrevistas sobre temáticas tales como las redes de solidaridad e interdependencia. Por otro lado, resulta importante mencionar que las diferencias de perfiles socioprofesional y de trayectoria familiar-individual son susceptibles de influir en el tipo de representaciones adoptadas y en el grado de permeabilidad a los discursos externos, pero también en el grado de objetivación sobre la posición ocupada en la jerarquía social. Nuestra hipótesis es que los beneficiarios se han apropiado de diversas maneras del programa, lo cual, por un lado, nos habla de un proceso pasivo de recepción, asimilación y moralización, pero, por el otro, se trata de un proceso activo de reinterpretación y movilización de los contenidos. Lo anterior depende de la posición en el espacio social que cada hogar ocupe y de los procesos de movilidad social en los cuales se encuentren inscritos.

Como primera medida, expondremos las dinámicas que nos hablan de una apropiación

12 Teniendo como objetivo ofrecer una visión más allá de la simple realidad económica, en el análisis se incluyen otras dimensiones tomadas de la obra de Pierre Bourdieu (2002), quien define las diferencias en el espacio social en función de la distribución de los capitales económico, social, cultural y simbólico. 
del programa, luego abordaremos las representaciones que se han articulado alrededor de este para, finalmente, exponer algunas de las estrategias e interpretaciones. En algunos casos, cuando lo amerite, la apropiación ira acompañada de su respectiva interpretación.

Apropiación. Existe una apropiación de las modalidades del programa. Las transferencias exigen a los beneficiarios el mantener un vínculo constante con la administración; estos poco a poco (algunos con grandes dificultades sobre todo en la zona rural) se adaptan a unas tecnologías ligadas a la recepción de las transferencias (cuentas bancarias, tarjetas Sim) y aprenden a hacer uso de los canales institucionales de información (call centers, oficinas locales). Asimismo, la asistencia a charlas, convenciones y encuentros de salud y convivencia se vuelve también parte de la cotidianidad de los beneficiarios, a lo que se le suma la experiencia adquirida en trámites y recolección de documentos necesarios para su continuación en el programa.

El habitus lingüístico de los beneficiarios se ha visto influenciado por los discursos externos, para lo cual son determinantes los encuentros y el rol de los asistentes sociales. Términos como "incentivo", "crecimiento y desarrollo", "vulnerabilidad" o "cobertura" han sido integrados al vocabulario de los beneficiarios con sus respectivas interpretaciones y usos. En el siguiente extracto se puede apreciar la interpretación de la palabra incentivo por una madre de un barrio de Cartagena cuando se le preguntó sobre los montos de la transferencia.
"Uno quisiera más, pero ellos dirán si te los dan o no te lo dan, yo digo que eso es como un incentivo como la palabra que ellos dicen, que te dan para que tú te esmeres para hacer las cosas y si te lo dan bien y sino, también" (ec1, Cartagena, 33 años, ama de casa, 5 años en el programa).

Esta adopción se evidencia en diferentes relatos, nuestra hipótesis es que la población categorizada como pobre se encuentra más expuesta a la adopción de discursos externos, para lo cual es determinante el bajo capital cultural y la posición social que los ubica en desventaja ante quienes emiten los discursos desde la autoridad que los inviste. En este caso, la transferencia es interpretada como un incentivo a la acción, a la búsqueda de oportunidades. En este extracto se aprecia también otro elemento que será tratado posteriormente: el de la no aprehensión de la transferencia como un derecho, como cuando la entrevistada dice "si te lo dan bien y si no, también". Teniendo como referente la apropiación del significado de la palabra incentivo, pasaremos a debatir la apropiación de la pobreza como asunto familiar e individual.

La superación de la pobreza ha sido interpretada por los beneficiarios como una cuestión que depende de la buena gestión que cada familia realice de la transferencia monetaria. De esta forma, la superación de la pobreza se presenta como una cuestión dependiente del esfuerzo personal y, por tanto, es de tipo individual. El siguiente extracto es interesante en la medida que la entrevistada maneja la representación de que las ayudas permiten la salida de la pobreza, pero luego se da una negociación de lo expuesto. 
"I: ¿ Y entonces crees que ese tipo de ayudas van a permitir que se acabe con la pobreza en Colombia?

ESJ28: Sí, si lo sabemos invertir [bastante segura].

I: ¿Entonces crees que de aquí a diez años las cosas van a cambiar?

[Lo piensa 05 segundos]

ESJ28: Mmm [Duda] No, porque... porque así como lo cogemos [el dinero] así mismo se nos va" (ESJ28, 26 años, artesana, rural, 5 ańos en el programa).

En el siguiente extracto se aprecia lo anterior, pero también otra idea a la que los PTC contribuyen desde sus modalidades: la exaltación de la mujer como mejores administradoras de los recursos familiares, relegando al hombre de la esfera doméstica.

"Para mí esos programas del Gobierno hay es que saberlos aprovechar, son buenos, son una ayuda en cualquier momento, uno tiene que saber aprovechar, administrar la plata, no esperar que el Gobierno venga a darle a uno las cosas, para mejorarle la calidad de la vida a los hijos, porque hay gente, sobre todo los papás, que no le dan la plata a los hijos sino que se la jartan en ron" (EC5, urbano, 38 años, 10 años en el programa, carrera técnica trabaja, como empleada doméstica).

En las entrevistas se indagó por el programa pero también por los problemas que afectaban a los territorios, esto generaba que las madres se vieran enfrentadas a ambas representaciones que manejan del Estado y de las autoridades locales; por un lado, la del
Estado benefactor que ayuda con ese tipo de programas y, por el otro, a la realidad de corrupción, clientelismo político y de olvido estatal como en la insuficiencia en la oferta pública. Lo que se pudo apreciar es que la situación adversa en cuanto a oferta pública de estos barrios, veredas o pueblos era de alguna forma normalizada y aceptada por tratarse de situaciones ya cotidianas.

De esta forma, las madres de familia manejan un doble discurso donde, por un lado, se busca resaltar los esfuerzos individuales de superación de la pobreza como las inversiones en los infantes y la buena administración de la transferencia. Pero este se ve opacado ante las restricciones que estos encuentran en su realidad, como por ejemplo, la necesidad de gasto inmediato, la falta de una oferta pública adecuada (sobre todo en el medio rural), la difícil inserción laboral, pero también por la realidad política-clientelar de los barrios y las veredas que habitan. Por esto, argumentamos que los recursos dispuestos pueden ser difícilmente convertibles en capacidades, lo que tiene como consecuencia que se tienda hacia una culpabilización del pobre por su condición.

Bajo este mismo esquema, nos interesa señalar la representación de la pobreza como un "espíritu", es decir, como disposición o actitud que diferencia a los pobres, entre quienes realizan esfuerzos por salir de la pobreza y quienes no lo hacen. Se busca así desligar el componente material de lo que sería un componente psicológico de la pobreza, pero también se convierte en una forma para algunos beneficiarios de reinterpretar su po- 
sición en el espacio social, no solo respecto a sus pares, sino también con respecto al resto de la sociedad. En el extracto siguiente podemos ver de manera parcial lo descrito por una mujer que vive en un barrio ilegal del oriente cartagenero, con formación técnica pero que trabaja en servicio doméstico. En el segundo caso, se trata de una madre líder que reinterpreta su situación y busca transmitir ese mensaje a sus pares.

"Aunque yo vivo aquí, yo no tengo mentalidad de pobreza, porque uno tiene que tener una mentalidad de riqueza, porque si tú vas con esa mentalidad de pobreza siempre va a ser uno pobre y ¡no!” (EC5, urbano, 38 años, 10 años en el programa, carrera técnica, trabaja como empleada doméstica).

"Así que yo le digo a la gente que uno es pobre de pronto, pero no de espíritu, por eso uno tiene que buscar lo que le toca $[. .$.$] que uno tiene que gestionar, ajá, para$ conseguir" (e35sJ, madre líder, Las Palmas).

La inversión en capital humano es vista como una obligación comprensible por las madres, la condicionalidad ha sido adoptada por los beneficiarios como una obligación obvia, y los controles necesarios a la recepción de la transferencia han pasado a formar parte de la cotidianidad de las madres de manera que el "buen uso" está asociado la inversión en estos componentes. El siguiente extracto es de una joven desplazada, hija de un líder de la comunidad, que terminó el bachillerato (su madre recibía FA) y comienza una formación técnica que luego abandona; su perfil permite una explicación más elaborada sobre la supe- ración de la pobreza, lo que nos habla de una reinterpretación; sin embargo, nos interesa señalar aquí que, para esta, la transferencia es de destino exclusivo para la inversión en capital humano.

“...yo digo que lo que te están dando es para... te colaboran con la alimentacion de tus hijos, ellos no te estan dando para que tú te superes, para poderme dar a mí para superar la pobreza tienen que darme trabajo que es lo que uno más hace pa superar la pobreza, lo que te están dando es una colaboracion para la alimentacion del bebé, que uno lo agredece porque uno sin trabajar, tiene esa colaboracion, le sirve esa ayuda" (E23c, urbano Cartagena, desplazada, 3 hijos).

Para algunas de las madres, el hecho de que no exista un control sobre el uso de la transferencia resulta incomprensible; estas, de alguna forma, se alinean con la manera en que tradicionalmente se ha manejado la ayuda a los pobres.

"Yo digo que no se hace un control para saber qué es lo que las madres hacen con eso, por lo menos aquí en el barrio cuando recibían esas platas, $\mathrm{xx}$ hacían paseos y [...] la platica de los nińos se la bebían los papás [...] yo digo que la mayor parte de esa plata no va para los niños, por lo menos yo lo cojo para él. [...] Eso es para que den un bono de estudio para comprar los cuadernos, así sí hay un mayor control” (E3C, urbano Cartagena, 32 años, formación técnica, 3 años programa).

Nos interesa ahora señalar las dinámicas de control como efecto social no deseado del programa, pues a pesar del libre uso de las 
transferencias como principio de los $\mathrm{PTC}^{13}$, el uso que las madres le dan a estas ha pasado a ser un elemento central del programa.

"I: ¿¿Alguna vez te has sentido señalada por recibir el subsidio?

EC7: De pronto me sintiera seńalada si lo cogiera para mí" (EC7, urbano, 27 años, ama de casa, antigua madre líder).

La clásica distinción entre el "buen pobre" y el "mal pobre" se da implícitamente en el discurso que desde la institucionalidad se transmite a los beneficiarios del programa en cuestión. Esta categorización opera para distinguir aquellos que utilizan los recursos del programa para uso exclusivo de los niños, pero también para aquellos que aplican hábitos comportamentales considerados deseables (cuidado en salud constante de los niños, acompañamiento escolar), una redención a través del trabajo, así como un comportamiento moral asociado a una racionalidad en las decisiones.

En las entrevistas con los funcionarios locales se exaltan los casos de niños con buen desempeño escolar o las familias que gracias al subsidio han logrado cambiar su situación, al mismo tiempo que se señalan o exaltan como malos ejemplos a aquellos que utilizan los recursos para otros fines. Estos últimos representados como irresponsables en su com- portamiento sexual-reproductivo, asociado a lugares comunes como el alcoholismo, la prostitución y la pereza, preso del "espíritu de la pobreza”.

Las madres líderes han adoptado como propia la tarea de transmitir, no solo las informaciones concernientes al programa, sino también los discursos que emite la institucionalidad. Estas afirmaciones se vuelven más pertinentes para el caso de comunidades de pequeńa talla y en medio rural, donde el grado de cohesión social tiende a ser más importante (salvo en los casos de comunidades desintegradas por el conflicto armado).

El siguiente extracto nos sirve para ilustrar cómo las madres líderes han adoptado una posición de defensa de lo público, aun cuando su trabajo es de carácter voluntario, pero también la manera en que las reuniones se vuelven lugares de intercambio de información sobre comportamientos deseables.

\begin{abstract}
"A veces hay unas [madres] que me preguntan, ¿XX cuándo es que van a pagar? Y yo les digo, aja y ¿tú pa quién trabajas?, entonces yo les digo que no estén pendiente de la plata, por lo menos en el proceso de verificación están los controles, entonces cuando los niños ya pasan del ańo, cuando ya no es obligatorio, yo les digo que los sigan llevando a los controles de oídos, de los ojos, de odontología [...] y les digo que si van pal centro váyanse con su pantalosito limpio que así sea viejito uno va limpio, yo me las cojo así, sobre todo cuando hago las reuniones, de pronto me dicen sapa por detrás,
\end{abstract}

13 "Los programas utilizan principalmente transferencias monetarias de libre uso ya que se considera que los propios hogares son los que mejor uso pueden hacer de dichas transferencias sobre la base de sus preferencias" (Ceccinni y Madariaga, 2011). 
pero bueno yo quiero es ayudarles" ( $\mathrm{E} 35 \mathrm{sJ}$, rural centro poblado, madre líder, desempleada [antes ICBF ], 3 años en el programa).

Las madres líderes constituyen un perfil particular dentro de los beneficiarios descritos (Ayola Betancourt, 2015), pues aunque estas son transmisoras y se encargan de mantener un orden (que evidentemente se escapa de sus posibilidades), también hacen parte de las comunidades que habitan y estas saben movilizar esta doble pertenencia a fin de gestionar recursos para sus comunidades y consolidar un capital social.

Para terminar, se puede decir que el programa contribuye a la expansión de una visión moralizadora que busca inculcar valores a los pobres con el objetivo de "corregirlos". Pero lo anterior no es exclusivo del programa, ha formado parte de la manera en que las sociedades occidentales han buscado corregir a sus elementos "patológicos", sea a través del trabajo, del disciplinamiento, de la sumisión a la religión o a un ideal de higiene (Álvarez, 2005). El programa no es entonces responsable de esta dinámica, aunque sí contribuye a través de los valores transmitidos a reforzar el conjunto de creencias y representaciones sobre quién merece la asistencia.

Representaciones sociales. A continuación se abordarán algunas representaciones sociales que se han erigido en torno al programa. Las representaciones son formas en que la realidad es aprehendida y explicada por los individuos, toman valor de realidad concreta y pueden orientar las conductas de los individuos; estas se convierten en fenómenos sociales en la medida en que son compartidas por un grupo social. La investigación realizada nos indica que existen dos tipos de representaciones sociales que podrían ser asociadas a imaginarios, pero que toman valor de realidad concreta y se encuentran principalmente poniendo en cuestión el programa como artífice de derechos.

Primero, en las entrevistas realizadas emerge continuamente una representación sobre el programa, la de Familias en Acción como un regalo que otorga el Gobierno (sí existe una asociación con la figura del Ejecutivo) a las familias pobres, una "ayudita" que permite paliar las necesidades de las familias, la cual es aprehendida como un regalo o un favor y afirma la representación del Estado paternalista, personalista y caritativo.

"Para mí eso son [refiriéndose a FA], digo yo, ayudas que el Gobierno nos brinda para nuestros hijos, yo digo que esos son regalos, aunque tenemos derecho, pero con tantas cosas que pasan” (EsJ28, 26 años, rural vereda, 5 años en el programa).

“Yo recibo por mi bebé 120 mil pesos, pa' mí que eso está bien, lo que es regalado uno lo recibe así sean $20 \mathrm{mil}$ pesos yo los recibo porque no los he trabajado" (EC13, Cartagena, 26 años, ama de casa, 1 año en el programa).

Si bien no se puede decir que todos los beneficiarios adopten esta representación, pues en la medida que estos conocen más sus derechos o se encuentran dentro de procesos de reivindicación colectiva esta representación tiende a variar, sin embargo, se puede decir que en este punto se llega a una "saturación de la información”, es decir, que en el universo 
de estudio es recurrente esta representación manifestada de manera verbal o no verbal.

Las representaciones son vectores de las conductas individuales. La representación de FA como un regalo implica que las madres de familia adopten una postura de agradecimiento para quienes presumen como responsables, lo cual es ante todo asociado al Ejecutivo. Teniendo en cuenta que en Colombia la asistencia a los pobres ha sido una tarea históricamente delegada a la iglesia católica y a la caridad privada hasta entrado el siglo xx. De alguna forma, la política de asistencia social sigue estando condicionada por la manera en que históricamente el Estado y la sociedad se han relacionado con los pobres en el país (Castro, 2007) es decir, por los esquemas de ayuda basados en la caridad.

Además, volviendo al plano normativo, al derecho se está accediendo más por la comprobación de la calidad de pobre -hoy objeto de la solidaridad internacional- que por la condición de ciudadano en dificultad objeto de la solidaridad estatal.

Esta es una representación que indudablemente no coincide con el esquema de asistencia basada en el derecho de igualdad de los ciudadanos, tal como lo postula la Carta Política (art. 13), ni con lo establecido por la Ley 1532 de 2012 que dota al programa de una estabilidad jurídica y lo proclama como derecho adquirido de los beneficiarios.

Lo anterior está relacionado, por ejemplo con que estos consideren el monto de la transferencia como "lo justo" y que tampoco se entienda este como derecho de los niños ni de las familias. Los extractos de entrevistas que presentamos a continuación nos sirven para ilustrar este punto sobre el monto y la representación del subsidio como un regalo.

"Uno quisiera más, pero ellos dirán si te los dan o no te lo dan [...] Por la cantidad de dinero, ¡claro si me dan más gracias a Dios! Pero digo que eso debe ser lo justo" (Ec1, Cartagena, 33 años, ama de casa, 5 ańos en el programa).

A pesar de esto, el monto puede ser puesto en cuestión -sobre todo el incentivo otorgado a los niños que cursan primaria (20 mil pesos bimensuales) - sobre todo por las familias que presentan menor dependencia económica. El caso siguiente es el de una mujer ama de casa y artesana, donde el proveedor de la familia cuenta con ingreso estable.

“...el único pańito de agua que viene son los pagos de FA, cuando vienen esas platicas eso es una bendición, y así como decía yo, eso es relativo, porque a un niñito de primaria que le dan 15 mil pesos, también se ve tan, ay, tan chiquitico, pero bueno, igual eso es regalado" (EsJ23, San Jacinto, urbano, 38 años, artesana, 6 años en el programa).

El manejo de esta representación incide en que ante retrasos en los pagos, incoherencias en los montos transferidos o en la actualización de datos, los beneficiarios prefieran no quejarse ante la entidad correspondiente. Este fenómeno ha sido estudiado en un contexto diferente por Warin (2012) quien analiza el no acceso a los derechos sociales en el caso francés (non recours, non-take up of social rights) considerándolo un asunto que pone en cuestión la pertinencia de las políticas públicas. Si se recuerda el primer extracto presentado, 
donde la entrevistada manifestaba que recibir o no la transferencia le era indiferente, a continuación se cuestionaba puntualmente sobre esta tendencia.

"I: ¿alguna vez has tenido problemas con los pagos?

ESJ28: Sí, antes, una vez me vino por siete mil y otra vez no llegaron $[\ldots]$, pero yo nunca metí el reclamo.

I: ¿Y eso por qué, mucho papeleo?

ESJ28: no, no sé (risas) no... (silencio)” (esj28, 26 años, rural vereda, 5 años en el programa).

Lo anterior, entonces, es sintomático de una debilidad de la noción de ciudadanía, sobre lo cual incide que sea un derecho que se otorga bajo ciertas condiciones pero también de las modalidades con las que el programa se puso en marcha en los primeros ańos cuando estuvo asociado a la figura del Ejecutivo y al voluntarismo político que de este se derivaba (Pulido, 2013). Esta es otra representación que se hizo evidente en las entrevistas, la cual no será abordada.

La reinterpretación. Sin embargo, lo expuesto anteriormente no quiere decir que los beneficiarios adquieran un papel pasivo en la adopción de discursos externos; de hecho, estos hacen uso de diferentes estrategias de apropiación, las cuales nos hablan de un proceso de interpretación activo de los componentes de la asistencia y de los instrumentos de focalización.

Primero, los beneficiarios no se sienten estigmatizados por el hecho de recibir las ayudas, estos intentan no basar su identidad en su relación con la recepción de la transferencia ni, en general, con los programas de la asistencia social. Los sentimientos de incomodidad, pena, ruptura, señalamiento no parecen estar asociados a la transferencia. Esto fue abordado, por ejemplo, al interrogar sobre las condicionalidades que podrían afectar la estima como por ejemplo, las largas filas, congestión en las oficinas, dificultades en los pagos, etc. Lo observado nos indica que quienes son más dependientes de la transferencia obvian lo relativo a un señalamiento. En cuanto a las filas de cobro, se encuentra que quienes debutan procesos de distinción o de movilidad social, manifiestan utilizar estrategias para evitar el señalamiento como, por ejemplo, no hacer las filas de cobro los mismos días del pago o trasladarse a barrios con menor concentración de la población beneficiaria. Esto nos habla de un posicionamiento activo de apropiación por parte de algunas madres, lo cual está relacionado con la posición que el beneficiario tenga dentro del grupo de los pobres; en este caso se trata de una mujer que tiene un trabajo estable en una institución educativa como aseadora.

"Yo, en la cobrada nunca he tenido problema porque nosotros acá, de la zona esta, siempre buscamos la misma, ¡yo no! yo fui más inteligente, yo cobro es en Bocagrande, yo no hago fila, [...] donde no hay fila, no hay atraco y no hay nada, me voy en una moto" (EC8, Barrio Candelaria, Cartagena, 57 años).

Lo anterior lo explicamos por la particularidad que tiene la experiencia de la pobreza en el caso colombiano; para esto postulamos 
que esta se acerca al tipo de pobreza integra$\mathrm{da}^{14}$ en la tipología propuesta por Paugam (2013). En esta, los pobres son un grupo considerable de la población, por lo cual es una experiencia que no implica ruptura con el grupo social.

Segundo, encontramos como signo de la apropiación, no solo del programa, sino de la política de asistencia social en su integridad, las estrategias utilizadas por los beneficiarios para mantener su categorización como pobres ante las herramientas designadas de focalización. La focalización cada vez más restrictiva ha contribuido a la circulación entre los beneficiarios de estrategias para que estos puedan seguir siendo potenciales beneficiarios de la asistencia social.

La Ley 1532 inauguró la posibilidad de graduación del programa, es decir, que de comprobarse una mejora en la condición global del hogar, a estos se les otorga un periodo de gracia de transición pero, en efecto, estos podrían dejar de recibir las ayudas. Ante esto, los beneficiarios tienden a afirmarse como grupo: el de los "pobres", lo que preocupa principalmente a quienes tienen menos dependencia económica respecto a la transferencia.

"Yo pienso que igual aquí todos somos pobres, pero habemos personas que tenemos posibilidades, que están más cómodas que otras, pobreza hay, bastante, sobretodo pa los barrios, por allá para La Paz, uno por acá como que siempre como que más, pero eso no quiere decir que uno deje de ser pobre" (ESJ23, San Jacinto, urbano, 38 años, artesana, 6 años en el programa).

En las entrevistas surgen referencias a casos de personas que tienen la necesidad de la transferencia pero que no son focalizadas ni han alcanzado a entrar en las coberturas, lo que despierta sentimientos de solidaridad con estas. Algunas estrategias son movilizadas por los beneficiarios, en lo que tiene que ver con la focalización por el Sisben. Para ilustrar este punto presentamos estos dos extractos de entrevista:

“...hubo mucha gente que se quejó porque el puntaje estaba alto, sacaron a muchos, sí porque en la encuestas, por lo menos te preguntan qué estudias tú, tal, qué eres tú, tal, [...] todo eso lo van incluyendo y lo arroja el sistema, cuando a mí me lleguen a hacer encuestas, ¿̧hasta qué curso llegaste? ¡pan! hasta octavo. Octavo, porque uno, a mí me lo explicó un amigo que es el que hace las encuestas, me dijo, nunca cuando te vayan a hacer una encuestas digas [realmente] hasta dónde llegaste [en tu formación escolar] [...] Porque el hecho de que yo tengo mi televisorcito, mi equipito, mis cositas, no es que yo tengo plata, uno se tiene que esmerarse con sacrificio" (EC5, urbano, 38 años, 10 años en el programa, carrera técnica, trabaja como empleada doméstica).

“...tienes que esconder ahora la neverita que compraste, que te sacrificaste, que pagaste con crédito y que no tenía

14 Algunos elementos del tipo de pobreza integrada son: la falta de presencia estatal se compensa con las solidaridades locales y tradicionales; la familia juega un importante rol como entidad que provee protección y reconocimiento, a su vez que permite la integración de estos al sistema normativo; el trabajo informal es la fuente de integración económica de los pobres. Desde el discurso que manejan con una focalización que es cada vez más restrictiva se busca evitar la aparición del polizón o free-rider. 
sino hielo y agua pa' que no te pongan más puntaje, ellos no miran el sacrificio con que esa nevera llega allí. Toca esconderla o mudarte para donde el vecino que esté peor que tú. $\mathrm{O}$ si compraste algo, decirle al vecino que te lo guarde" (Ec1, urbana, 33 años, 5 ańos en el programa, barrio Vistahermosa).

\section{CONCLUSIÓN}

El análisis del programa FA que hemos emprendido nos permite concluir que este es portador de una manera específica de concebir la pobreza, los beneficiarios han articulado representaciones y explicaciones en torno a los fenómenos de la pobreza como sujetos receptores e interpretativos, al mismo tiempo que se han apropiado de los instrumentos del programa como sujetos activos.

El enfoque por los instrumentos nos ofrece una herramienta analítica alternativa para el estudio de las políticas públicas, el cual nos ha permitido estudiar los instrumentos en su calidad de dispositivos normativos generadores de efectos sociales. Con este tipo de análisis se busca contribuir a la dinamización del debate sobre pobreza y desigualdad en el país, al integrar la visión de los actores directamente tocados por las políticas. Como principal resultado nos interesa destacar la percepción de la asistencia social como un favor que emana desde el Gobierno hacia los pobres del país. Este resulta sintomático de una relación que se perfila como desigual y contraria a los principios que rigen las democracias occidentales.

Nos interesa volver a señalar que el instrumento por sí solo no es articulador de la dinámica, sino que se inscribe en un contexto ideológico más amplio -que se puede resumir en la hegemonía de la economía de mercadoque inevitablemente lo condiciona. Los PTC constituyen una evidencia sobre cómo -bajo el marco de la lucha contra la pobreza- se ha dado una rearticulación de la base ideológica de la política social (Lautier, 2013).

Para Pierre Muller (citando a Habermas, 1987 y Papadopolus, 1995), el análisis de políticas públicas es revelador de la manera en que las sociedades modernas se piensan a ellas mismas y buscan imponer un orden a través de su acción. Este argumento se articula con la sociología de la pobreza (Paugam, 2013), la cual interpreta las acciones de regulación de la pobreza como un elemento explicativo de cómo la sociedad se piensa a ella misma. De esta forma, el estudio de las formas de regulación de la pobreza en el país plantea interrogantes no solo para la comunidad académica, sino también para el conjunto de la sociedad sobre las armas con que esta planea enfrentarse a un escenario de posconflicto.

\section{REFERENCIAS}

Álvarez, S. (2005). La producción social de la pobreza. En: Los discursos sobre pobreza y el desarrollo humano. Buenos Aires: Lumen Humanitas.

Álvarez, S. (2013) Sumario Voces abiertas. La dignidad de los nadies, 4 (22).

Ayola Betancourt, V. (2015). La régulation de la pauvreté en Colombie au prisme de la théorie des liens sociaux : le cas de Familias en Acción. (Tesis inédita de maestría). École des hautes Études en Sciences Sociales, Paris, Francia.

Banerjee, A.y Duflo, E. (2012). Repenser la pauvreté. Paris: Editions du Seuil. 
Becker G. S. (1964). Human Capital. A Theoretical and Empirical Analysis, New York: Columbia University Press for the National Bureau of Economic Research.

Bourdieu, P. (2002). Condición de clase y posición de clase. Revista colombiana de sociología. 7(1), 119-141.

Castro Carvajal, B. (2007). Caridad y Beneficencia. El tratamiento de la pobreza en Colombia, 18701930. Bogotá D.C.: Universidad Externado de Colombia.

Ceccinni, S. y Madariaga, A. (2011). Programas de Transferencias Condicionadas. Santiago de Chile: CEPAL.

Debonneville, J. y Díaz, P. (2011). Innovation, diffusion et rôle des idées: une lecture internationale des Conditional Cash Transfers aux Philippines. En Contribution à la séance thématique RTG Protection sociale, politiques sociales et solidarités: Innovation et expérimentation: acteurs, territoires et dispositifs. Congrès 2011 de l'AFs.

Gabas, J. J. y Laporte, C. (2012). La pauvreté dans l'agenda des Nations Unies. Ceriscope Pauvreté. Recuperado de http://ceriscope.sciences-po. $\mathrm{fr} /$ pauvrete/content/part4/la-pauvrete-danslagenda-des-nations-unies

Giraldo, C. (ed.). (2013). Politica social contemporánea en América Latina. Bogotá D.C.: Universidad Nacional de Colombia, Ed. Desde Abajo.

Holzmann, R. y Jorgensen, S. (2000). Gestion du Risque sociale: Théorique cadre de la Protection sociale. En Discussion Paper Series, no. SP 6. Banque mondiale : Washington, DC.

Jobert, B. (1994). Le tournant néo-libéral en Europe. Idées et recettes dans les pratiques gouvernementales. Paris:L'harmattan.

Jodelet, D. (1989). Les Représentations Sociales. Paris: Presses Universitaires de France.
Lascoumes, P. y Le Galès, P. (2004). Gouverner par les instruments. Paris: Presses de Science Po.

Lascoumes, P. y Simard, L. (2011). L'action publique au prisme de ses instruments. Revue française de science politique, 61, 5-22.

Lautier, B. (2010). Les politiques sociales à l'épreuve des réformes de marché. Revue internationale de politique comparée, 17 (3), 23-55.

Lautier, B. (2013). Les сcтp en Amérique Latine: Entre modélisation internationale et conjonctures politiques nationales. Revue Tiers Monde (214).

Lo Vuolo, R., Barbeito, A., Pautassi, L. y Rodríguez, C. (2004). La pobreza de las politicas contra la pobreza. Buenos Aires: Centro Interdisciplinario para el Estudio de las Políticas Públicas.

Merrien, F. X. (2013). La protection sociale comme politique de développement: Un nouveau programme d'action international. International Development Policy. 4(2), 68-88.

Morley, C. (2003). From Social Assistance to Social Development: targeted education subsidies in developing countries. Washington: Center for Global Development and IFPRI.

Muller, P. (2000). L'analyse cognitive des politiques publiques: vers une sociologie politique de l'action publique. Revue française de science politique, 50 (2), 189-208.

Noël-Roth, A. (2009). La evaluación de políticas públicas en Colombia: una mirada crítica a partir de las prácticas evaluativas oficiales de los programas de la Red de Apoyo Social. Revista del CLAD Reforma y Democracia, 45.

Paugam, S. (2013). Les formes élémentaires de la pauvreté. Paris: PuF.

Pinson, G. y Sala-Pala, V. (2007). Peut-on vraiment se passer de l'entretien en sociologie de l'action publique? Revue française de science politique, 57, 555-597. 
Pulido, C. (2013). Los programas de transferencias condicionadas en América Latina, el caso de Familias en Acción. (Tesis inédita maestría) Facultad de Derecho, Ciencias Políticas y Sociales, Universidad Nacional de Colombia, Bogotá.

Rawlings, L. (2004). A new approach to social assistance: Latin-American's experience with conditional cash transfer programs. International Social. Rev. 58, 133-161.
Sánchez, W. (2004). Análisis crítico del discurso: una aproximación. Bogotá: Pontifica Universidad Javeriana.

Sen, A. (1999). Development as freedom. New York: Oxford University Press.

Simmel, G. (2011). El pobre. Barcelona: Ediciones Sequitur.

Warin, P. (2012) Le non-recours aux droits. SociologieS. Recuperado de http://sociologies.revues. org/4103 